\title{
El tabaquismo: primera causa prevenible de muerte en el mundo
}

Es de sobra conocido que el tabaco constituye la primera causa de muerte prevenible a nivel mundial.

Se sabe que la mitad de quienes tienen el hábito de fumar mueren de forma prematura por enfermedades ligadas con el tabaco, en particular de tipo cardiovascular y respiratorio. El tabaquismo, triplica la presentación o complicación de las enfermedades cardiovasculares, y aumenta en 4 veces la posibilidad de padecer arteriosclerosis. La enfermedad pulmonar obstructiva crónica, condición que puede ser fatal, es 10 veces más frecuente en fumadores que en no fumadores, y el tabaquismo es probablemente responsable del $90 \%$ de las enfermedades respiratorias crónicas y de las muertes atribuidas a enfermedad pulmonar obstructiva crónica.

También se ha evidenciado que incrementa de manera importante la aparición de numerosos tipos de tumores, significativamente los de boca, faringe, laringe, tráquea, bronquios y pulmón.

Es preciso consignar el daño producido a un numeroso grupo poblacional, mediante la exposición pasiva de no fumadores, ya que éstos también pueden sufrir muchas de las consecuencias citadas.

De acuerdo con la Organización Mundial de la Salud, los niños y los adolescentes están en alto riesgo de iniciarse en el tabaquismo. Entre los 18 y los 25 años, un promedio del $23 \%$ al $24 \%$ de los individuos fuma, y hay un incremento en el género femenino.

El impacto del consumo del tabaco en los presupuestos de salud es tan grande, que más de cuarenta estados de los Estados Unidos de Norteamérica han demandado a la industria del tabaco, con el objeto de recuperar en parte el gasto económico destinado al tratamiento de enfermedades relacionadas con el tabaquismo.

Está demostrado que la nicotina produce adicción y cumple todos los criterios para convertirse en una droga que induce a la dependencia: ocasiona tolerancia y dependencia física, y cuando se suspende, se producen síntomas de abstinencia que varían en cada persona.

La Academia Nacional de Medicina de Costa Rica, por tanto, desea manifestar su firme posición en contra del tabaquismo.

Dr. Claudio Orlich Carranza

Presidente Academia Nacional de Medicina

ISSN 0001-6002/2004/46/4/170

Acta Médica Costarricense, (O2004

Colegio de Médicos y Cirujanos 